\title{
Erratum
}

Med Klin Intensivmed Notfmed 2020 · 115:237-238 https://doi.org/10.1007/s00063-019-00614-w Online publiziert: 28. August 2019 (c) Springer Medizin Verlag GmbH, ein Teil von Springer Nature 2019
K. Rygiel' $\cdot$ R. Fimmers ${ }^{2} \cdot$ S. Schacher ${ }^{3} \cdot$ H. Dormann ${ }^{4} \cdot$ I. Gräff ${ }^{1}$

${ }^{1}$ Interdisziplinäres Notfallzentrum, Universitätsklinikum Bonn, Bonn, Deutschland

${ }^{2}$ Institut für Medizinische Biometrie, Informatik und Epidemiologie, Universitätsklinikum Bonn, Bonn, Deutschland

${ }^{3}$ Zentrale Notaufnahme, Evangelisches Krankenhaus Köln Kalk, Köln, Deutschland

${ }^{4}$ Zentrale Notaufnahme, Klinikum Fürth, Fürth, Deutschland

\section{Erratum zu: Ältere Notfallpatienten in der zentralen Notaufnahme}

\section{Eine Kennzahlenauswertung auf Basis des DIVI-Notaufnahmeprotokoll}

\section{Erratum zu:}

Med Klin Intensivmed Notfmed 2019

https://doi.org/10.1007/s00063-019-

0595-2

Der ursprüngliche Artikel wurde korrigiert. In der zunächst veröffentlichten Online-Version waren die Legenden der Abb. 2 und 3 nicht vollständig. Bitte beachten Sie die folgenden ergänzten Fassungen:

Abb. 2 Prozentualer Erreichungsgrad der vorgegebenen MTS-Zeiten. Angegeben sind die prozentualen Erreichungsgrade der vorgegebenen maximalen Wartezeiten bis zum Arztkontakt mit 95\%Konfidenzintervallen in den MTS-Stufen „Blau“, „Grün“, „Gelb“, „Orange“ und "Rot", unterteilt nach Altersklassen. Innerhalb der MTS-Stufen sind die Werte für die fünf Altersklassen angezeigt (links beginnend 16-64, dann 65-70, dann 71-80, dann 81-90 und >90 Jahre). Es zeigt sich, dass mit zunehmendem Alter der Erreichungsgrad innerhalb der Triagestufen steigt. Überschreitungen in der MTS-Kategorie „Rot" (Arztkontakt sofort) können durch ein zeitverzögertes Setzen des "Timestamps Arztkontakt“ im Computersystem entstanden sein. Denkbar ist dies, wenn z.B. alle personellen Ressourcen primär in der unmittelbaren Patientenversorgung gebunden sind

\begin{abstract}
Abb. 3 Wartezeiten bis zum ersten Arztkontakt. Dargestellt sind die mittleren Wartezeiten bis zum Arztkontakt mit der Angabe von 95\%-Konfidenzintervallen in den MTS-Stufen „Blau“, „Grün“, „Gelb", „Orange“ und „Rot“. Innerhalb der MTS-Stufen sind die Werte für fünf Altersklassen angezeigt (links beginnend 16-64, dann 65-70, dann 71-80, dann 81-90 und >90 Jahre). Es zeigt sich, dass mit zunehmendem Alter die Arztkontaktzeit innerhalb der Triagestufen abnimmt
\end{abstract}

\section{Korrespondenzadresse}

PD Dr. med. I. Gräff, DESA

Interdisziplinäres Notfallzentrum, Universitätsklinikum Bonn

Sigmund-Freud-Str. 25, 53105 Bonn,

Deutschland

ingo.graeff@ukbonn.de
Die Online-Version des Originalartikels ist unter https://doi.org/10.1007/s00063-019-0595-2 zu finden. 
Hier steht eine Anzeige.

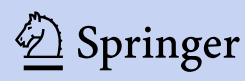

